Ann. Zootech., I975, 24 (I), 43-57.

\title{
INFLUENCE DE LA SUBSTITUTION DES PROTÉINES DU LAIT PAR DES PROTÉINES DE POISSON SUR QUELQUES CARACTÉRISTIQUES DU CONTENU GASTRIQUE CHEZ LE PORCELET SEVRÉ A 12 JOURS
}

\author{
B. SÈVE et J.-P. LAPLACE*
}

avec la collaboration technique de Anne-Marie Mounier, C. Germain et A. Lapanouse

Station de Recherches sur l'Élevage des Porcs,

* Laboratoire de Physiologie de la Nutrition, Centre national de Recherches zootechniques, I. N.R. A., 78350 Jouy en Josas

\section{RÉSUMÉ}

Une fistulation gastrique en région fundique a été réalisée chez 19 porcelets de race Large White sevrés à l'âge de $\mathrm{I} 2$ jours, opérés à 14 jours environ et d'un poids moyen de $4 \mathrm{~kg}$. Le $\mathrm{pH}$ intragastrique a été enregistré in vivo, durant des périodes diurnes de $7 \mathrm{~h}$. Des prélèvements de contenu gastrique, effectués avant et $\mathrm{I} h$ après les 2 principaux repas, ont permis la mesure du $\mathrm{pH}$ et de l'activité peptique des contenus homogénéisés. La teneur en matière sèche du contenu frais et la teneur en azote du contenu sec de l'estomac ont été mesurées sur l'homogénat et les fractions solide et liquide séparées par filtration.

Selon que, dans l'aliment utilisé, $4^{8} \mathrm{p}$. Ioo des protéines de lait sont ou non remplacées par des protéines solubles de poisson, une configuration particulière des courbes d'évolution du $\mathrm{pH}$ in vivo est observée. L'activité peptique, croissante avec l'âge, n'est pas affectée par la composition de l'aliment. La répartition de la matière sèche et cle l'azote entre les phases solide et liquide du contenu gastrique $\mathrm{I} h$ après l'ingestion d'aliment à base de lait permet d'évaluer à $70 \mathrm{p}$. Ioo la proportion d'azote immobilisée dans la phase solide contre $30 \mathrm{p}$. roo seulement pour l'aliment comportant des protéines solubles de poisson. L'importance de ces résultats est discutée en relation avec l'amélioration des performances de porcelets sevrés précocement.

\section{IN'TRODUCTION}

La pratique de l'allaitement artificiel du porcelet implique le respect de la physiologie digestive particulière de cet animal. Celle-ci se caractérise par un développement médiocre des sécrétions digestives, aussi bien gastriques que pancréatiques et intestinales (Lewis et al., I957; HARTMAN et al., I96I). De plus, comme chez tous les jeunes mammifères, l'estomac du porcelet semble posséder une activité 
coagulante vis-à-vis de la caséine du lait de truie. Lorsque le Porcelet reçoit du lait de vache plus riche en caséine, cette activité se traduit par la formation dans son estomac d'un coagulum dense (White et al., I969) alors que le lait de truie ne fait que floculer (BRAUDE et al., I958).

Le décours de l'évacuation gastrique du veau allaité et en particulier le rythme d'évacuation des fractions glucidique, lipidique et azotée du contenu gastrique sont largement conditionnés par le phénomène de coagulation (MATHIEU, I968). Cette influence est également très nette chez le Porcelet (HIL, L, Noakes et LowE, I969). L'évacuation gastrique de l'azote et des lipides (mais non des glucides) est considérablement retardée dans le cas du porcelet alimenté au lait de vache par rapport au lait de truie.

Dans l'état actuel de nos connaissances, la recherche d'un aliment pouvant se substituer au lait, après séparation de la mère, doit tendre à retrouver des conditions de milieu intra-gastrique (en particulier coagulation et transit des diverses fractions) aussi proches que possible de celles observées pour le lait de truie. L'objet de ce travail est l'étude comparative de l'influence de 2 aliments d'allaitement artificiel sur quelques caractéristiques physico-chimiques du contenu gastrique.

TABLEAU I

Composition centésimale des aliments

\begin{tabular}{|c|c|c|}
\hline & $A$ & $\mathrm{~B}$ \\
\hline 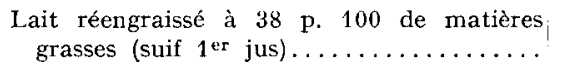 & 66 & 63 \\
\hline Lait écrémé $\ldots \ldots \ldots \ldots \ldots \ldots \ldots \ldots \ldots \ldots \ldots \ldots$ & 25 & - \\
\hline Concentré de protéines solubles de poisson $\left(^{*}\right)$ & 一 & 15 \\
\hline Maltodextrine $\left(^{* *}\right) \ldots \ldots \ldots \ldots \ldots \ldots$ & - & 18 \\
\hline Cérélose $\ldots \ldots \ldots \ldots \ldots \ldots \ldots \ldots \ldots$ & 5 & - \\
\hline Mélange minéral A ${ }^{(1)} \ldots \ldots \ldots \ldots \ldots \ldots$ & 1,70 & - \\
\hline Mélange minéral $\mathrm{B}\left({ }^{(2}\right) \ldots \ldots \ldots \ldots \ldots$ & $一$ & 1,75 \\
\hline $\begin{array}{l}\text { Mélange oligo-éléments }\left({ }^{3}\right) \text { vitamines, anti- } \\
\text { biotiques } \ldots \ldots \ldots \ldots \ldots \ldots \ldots \ldots \ldots \ldots \ldots \ldots \ldots \ldots\end{array}$ & 2 & 2 \\
\hline DL-méthionine $\ldots \ldots \ldots \ldots \ldots \ldots \ldots \ldots$ & 0,30 & 0,25 \\
\hline
\end{tabular}
(62480).

(*) Coopérative de traitement des produits de la pêche, Le Portel

(**) Promelka $1902 \mathrm{C}$, Société des Produits du Mais, Clamart (92140).

(1) Phosphate monocalcique : 0,1; phosphate bicalcique : 0,1 ; sel marin : 0,1 Lactosérum sec QSP : 1,70.

${ }^{2}{ }^{2}$ Phosphate bicalcique : 1,0 ; craie broyée : 0,3 ; sel marin : 0,1; Lactosérum sec QSP : 1,75.

$\left({ }^{3}\right)$ Iodure de potassium : 0,0002 ; sulfate de cuivre : 0,002 ; sulfate de zinc : 0,02 ; sulfate de cobalt : 0,001 ; sulfate de manganèse : 0,005 ; sulfate de magnésie : 0,100 ; sulfate de fer : 0,050 ; vitamine A $(50000 \mathrm{UI} / \mathrm{g})$ : 0,060 ; vitamine $\mathrm{D}(100000 \mathrm{UI} / \mathrm{g}): 0,015$; riboflavine : 0,001 Pyridoxine : 0,001 ; acide ascorbique : 0,008 ; concentrat de vitamine $B_{12}(100 \mathrm{mg} / \mathrm{kg})$ : 0,050 ; pantothénate de $\mathrm{Ca}: 0,004$; Concentrat de choline $(25 \mathrm{p} .100)$ : 0,$04 ;$ niacine : 0,$002 ; \alpha$-tocopherol : 0,$001 ;$ T.O. myxine $(8$ p. 100 : terramycine, 1 p. 100 oléandomycine) : 1,0; lactosérum sec QSP : 2,0. 


\section{MATÉRIEL, E'T MÉTHODES}

\section{Élevage}

Dix-neuf porcelets de race Large White ont été utilisés. Ils ont été sevrés à l'âge de $\mathrm{I} 2$ jours et placés en batterie, à raison de I porcelet par case, dans un local climatisé $\left(26^{\circ} \mathrm{C}, 60 \mathrm{p}\right.$. Ioo d'humidité). Ces porcelets ont reçu dès leur sevrage l'un ou l'autre des 2 aliments dont la composition figure dans le tableau I. Dans l'aliment A, la totalité des protéines est apportée par du lait de vache, alors que $4^{8} \mathrm{p}$. 1oo des protéines de l'aliment $\mathrm{B}$ proviennent d'un concentré de protéines solubles de poisson. Les animaux reçoivent deux repas par jour à $9 \mathrm{~h}$ et $\mathrm{I} 6 \mathrm{~h}$. L'aliment est proposé dans tous les cas sous la forme de granulés. La quantité d'aliment consommée est mesurée (tabl 3) pour l'heure qui suit chacune des deux distributions (quantité consommée au moment du prélèvement) et pour l'intervalle entre deux distributions (quantité ingérée avant la distribution-test). Les porcelets disposent d'un abreuvoir automatique qui ne permet pas le contrôle de la consommation d'eau.

\section{Opérations}

Tous ces porcelets ont été opérés aux âges et poids indiqués dans le tableau 2. L'intervention consiste en la réalisation, sous anesthésie générale (Penthrane ${ }^{\mathbf{R}}{ }^{(1)}$ appliqué au masque) d'une

\section{TABLEAU 2}

Caractéristiques individuelles des 19 porcelets opérés après seurage à l'âge de 12 jours

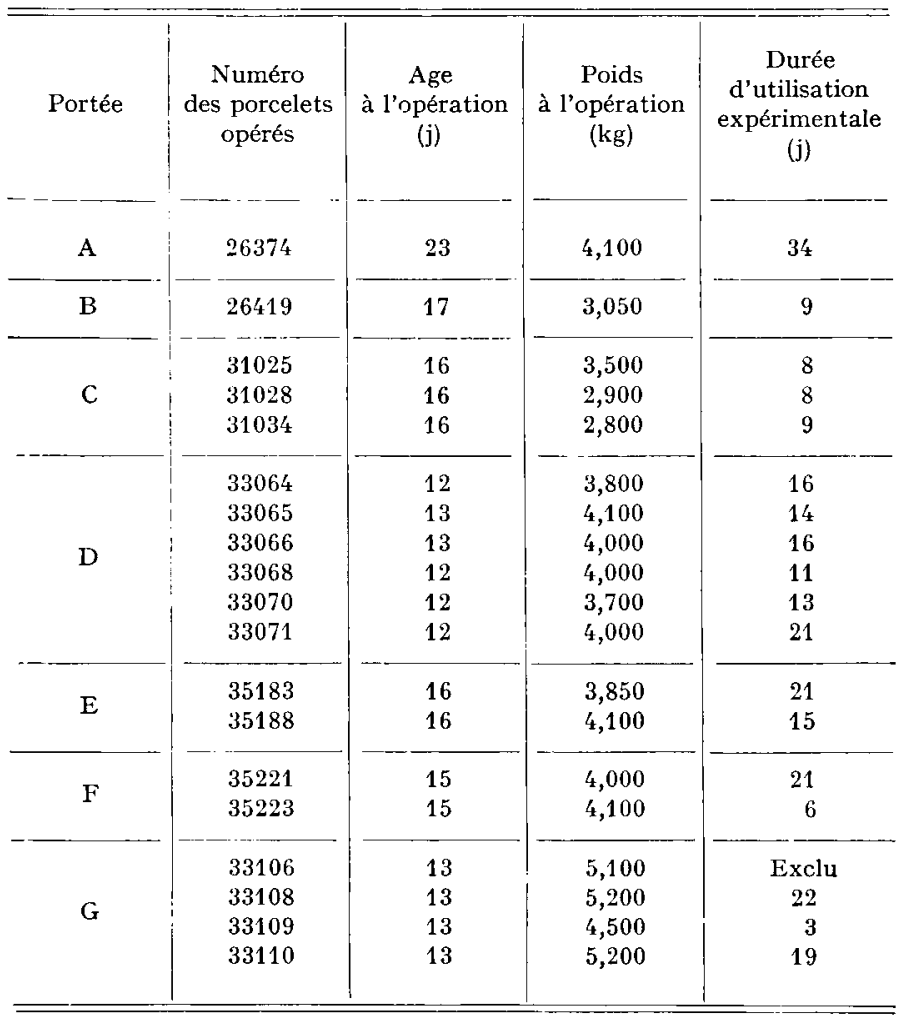

(1) Penthrane : méthoxyflurane, Laboratoires Abbott. 
fistulation gastrique. A la différence du précédent travail de l'un de nous (LAPLAcE, 1974) une canule droite réalisée en silicone médical, d'un diamètre intérieur/extérieur : 12,7/18 mm, est mise en place en région fundique, à mi hauteur de la grande courbure et au plus près de celle-ci sur la face viscérale de l'estomac. La canule est extériorisée au niveau de l'hypochondre droit (fig. I). Toutes les sutures (bourse au niveau gastrique, points en $x$ sur les plans péritonéal et

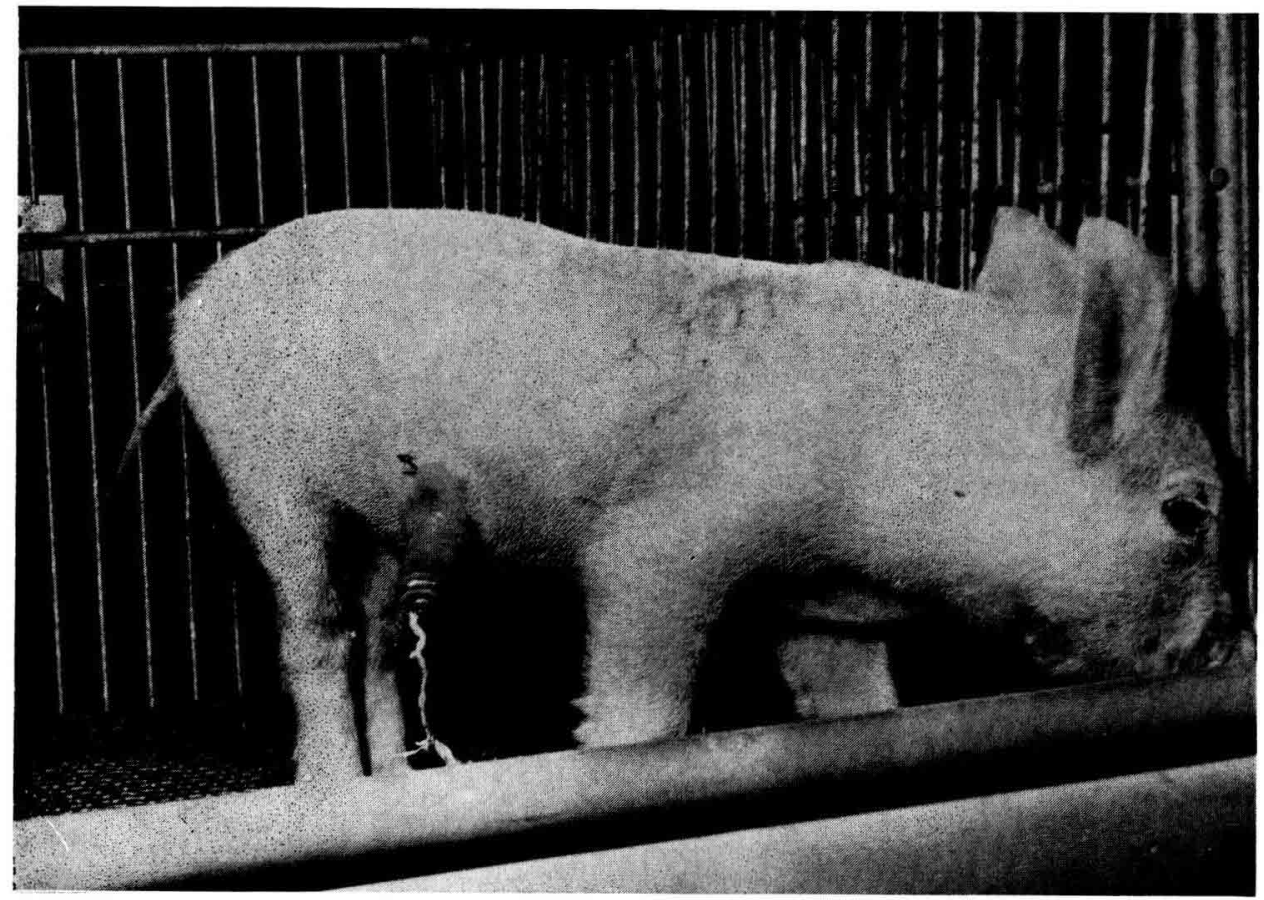

FIG. I. - Porcelet porteur d'une fistule gastrique permanente

musculaires, points séparés sur la peau) sont réalisées à la soie no o. Avant la fermeture de la brèche péritonéale, 500 ooo UI de pénicilline G sont déposées dans la cavité abdominale. Les porcelets sont réalimentés dès $24 \mathrm{~h}$ après l'opération. Ils n'ont fait l'objet d'enregistrements ou de prélèvements que 6 jours au moins après le sevrauge et 2 jours en moyenne après l'intervention. La durée de leur utilisation expérimentale a été dans la majorité des cas limitée par suite d'accidents (arrachage des canules accrochées dans les parois grillagées de la batterie). Chaque porcelet a été utilisé alternativement selon les jours, pour l'enregistrement continu du $\mathrm{pH}$ intragastrique in vivo ou pour des prélèvements du contenu gastrique.

\section{Enregistrements}

Pour les enregistrements continus, le Porcelet est installé dans une cage grillagée analogue à la case de sa batterie d'origine, avec eau et aliment à disposition, près des appareils nécessaires. Les électrodes stomacale et de référence, identiques à celles antérieurement utilisées chez des porcs plus âgés (LAPLACE, I974) ont été placées conjointement dans la canule gastrique. L'électrode stomacale $(4 \mathrm{~mm}$ de diamètre) est poussée de façon à émerger de l'orifice gastrique de la canule et à se trouver au sein du volume fundique. L'électrode de référence plus volumineuse, légèrement en retrait par rapport à la précédente, obture le corps de la canule en assurant l'étanchéité. Les 2 câbles des électrodes sont alors reliés à un pHmètre à lecture directe, lui-même connecté par l'intermédiaire d'un adaptateur à résistance variable au polygraphe d'enregistrement continu. Toutes les caractéristiques des appareils et des conditions techniques d'enregistrement ont été antérieurement décrites (LAPLACE, 1974). La durée des enregistrements est limitée à $7 \mathrm{~h}(9 \mathrm{~h}$ à $\mathrm{I} 6 \mathrm{~h})$, les distributions d'aliment étant effectuées, dans ces circonstances 
particulières, peu après $9 \mathrm{~h}$ et à $\mathrm{I}_{4} \mathrm{~h}$, de façon à disposer d'observations en période pré-et postprandiale.

Pour ne pas perturber les porcelets par des manipulations trop fréquentes, le nombre de prélèvements a été limité à deux par jour, avant et une heure après le début d'un des deux repas. La mesure du $\mathrm{pH}$ était faite sur du contenu brut immédiatement avant congélation de l'échantillon en vue des dosages biochimiques ultérieurs.

\section{Dosages biochimiques}

La moitié de l'effectif des échantillons correspondant à chaque temps de prélèvement a été filtrée, après décongélation, sur fibre de cellulose de façon à séparer la fraction solide de la fraction liquide du contenu gastrique. Les échantillons restants ont été homogénéisés par broyage. La matière sèche et les protéines ont été dosées dans chaque fraction (solide et liquide) ainsi que dans les contenus homogénéisés. Le dosage de l'activité peptique du contenu à pH 2 a été réalisé sur les contenus homogénéisés après dilution appropriée selon la méthode classique de ANson (I938). Le substrat utilisé était une solution d'hémoglobine purifiée (1) à 2,5 p. Ioo, le temps d'incubation à $37^{\circ} \mathrm{C}$ était de I $_{5} \mathrm{mn}$, et le calcul de l'activité était effectué selon la formule suivante :

A $280^{*} \times$ I 000

Temps $\times$ poids d'échantillon homogénéisé utilisé

* Taux d'absorption, à Ia longueur d'onde de $280 \mathrm{~m} \mu$, mesurant l'apparition des acides aminés aromatiques dans l'hydrolysat filtré.

\section{RÉSSULTATS}

\section{I. - Évolution du pH enregistré in vivo}

Le $\mathrm{pH}$ enregistré dans l'estomac du porcelet, le matin avant la première distribution d'aliment, est toujours compris entre 2,0 et 3,0 chez les animaux recevant l'aliment $\mathrm{A}$, et entre $\mathrm{I}, 5$ et 2,5 chez les porcelets nourris à 1 'aliment $\mathrm{B}$.

Par rapport à ces valeurs initiales, l'ingestion du premier repas $(9 \mathrm{~h})$ d'aliment $\mathrm{A}$ (4I $\mathrm{g}$ en moyenne) provoque $2 \mathrm{mn}$ après le début de ce repas une rapide augmentation du $\mathrm{pH}$ qui atteint une valeur comprise entre 5,0 et 6,0. Cette élévation est réalisée pour $80 \mathrm{p}$. Ioo de son amplitude en l'espace de 2 à $3 \mathrm{mn}$, et est totalement développée en 20 à $30 \mathrm{mn}$. Au-delà de cette valeur maximum, le $\mathrm{pH}$ décroît très

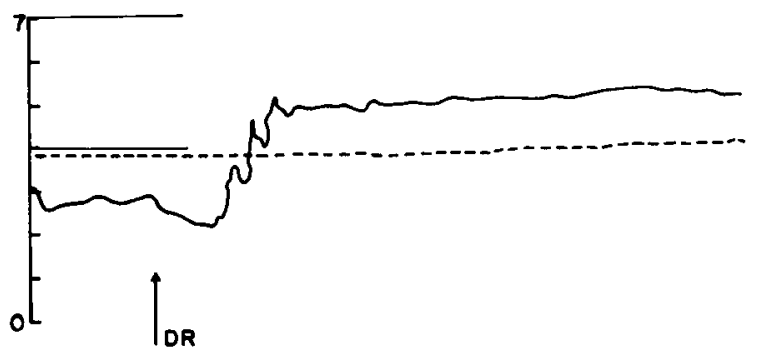

FIG. 2. - Evolution du pH intragastrique lors du repas du matin (-) et de l'après-midi (-..-) chez un porcelet âgé de 25 jours, recevant l'aliment $A$ $\mathrm{DR}$ : début du repas

(1) Worthington. 

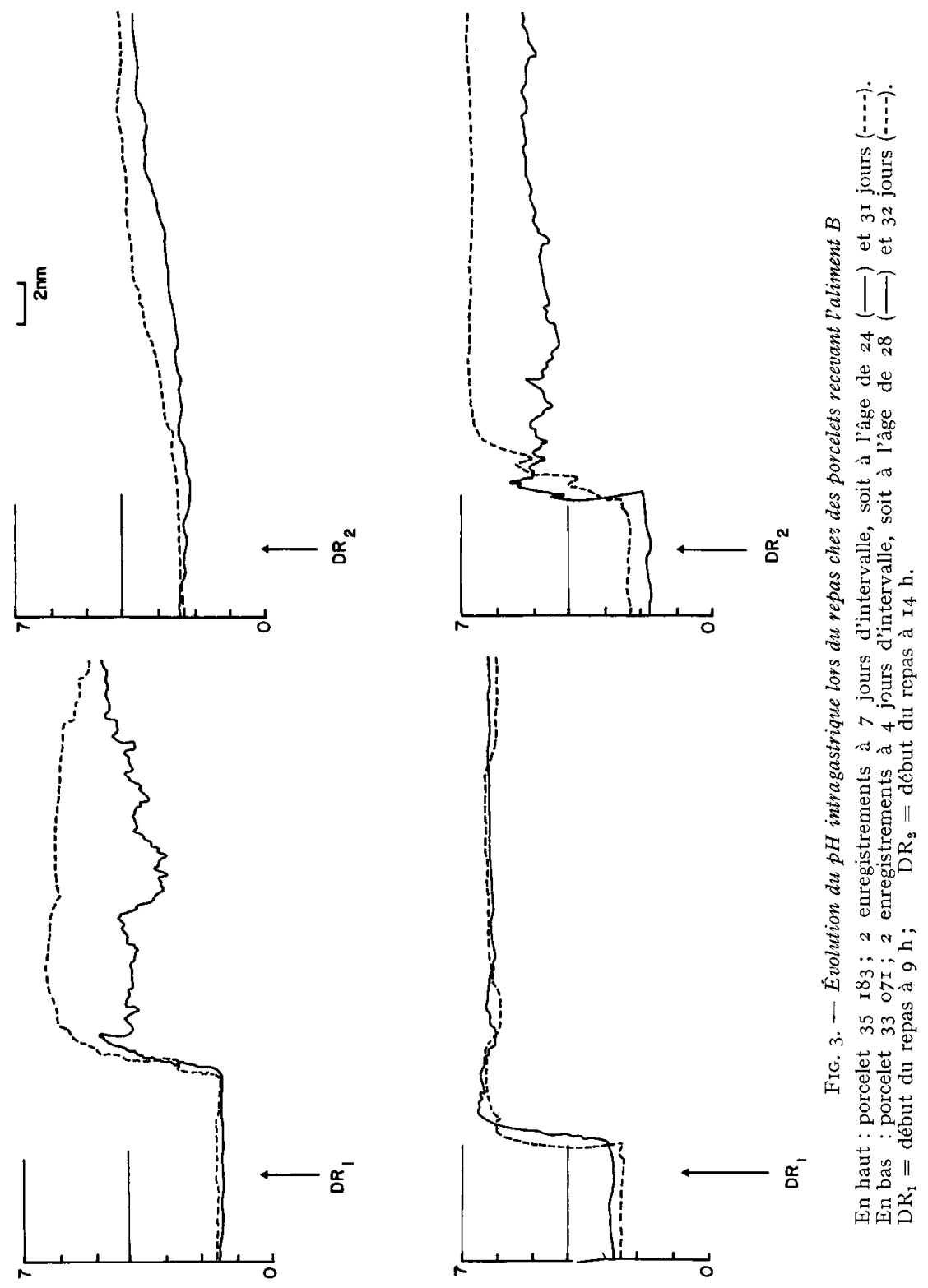

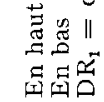


progressivement jusqu'à une valeur moyenne de 4,0 en l'espace de I20 à I70 mn. La courbe de $\mathrm{pH}$ reste alors stable jusqu'à la seconde distribution ( $\mathrm{I}_{4} \mathrm{~h}$ ). Le repas consécutif à cette nouvelle distribution d'aliment A (39 $\mathrm{g}$ ingérés en moyenne) ne provoque jamais de modification du tracé qui reste stable dans la gamme de pH 3,9-4,3 (fig. 2).

La distribution d'aliment $B$ conduit selon les animaux à 2 types de courbes (fig. 3). Ces caractéristiques des enregistrements sont des particularités individuelles, chaque porcelet conservant au cours de sa "vie expérimentale " un même type stable d'évolution. Pour le premier type, on observe une élévation du $\mathrm{pH} 5 \mathrm{mn}$ après le début du repas $(9 \mathrm{~h}, 5 \mathrm{I} \mathrm{g}$ ingérés en moyenne). Cette augmentation amène en l'espace de $2 \mathrm{mn}$ le $\mathrm{pH}$ à une valeur couramment comprise entre 5,0 et 6,0 (avec des extrêmes à 4,0 ou 6,5 ). La courbe de $\mathrm{pH}$ amorce, après un plateau durant 40 à $60 \mathrm{mn}$, une décroissance d'une amplitude de $\mathrm{I}, 5$ à 2 unités $\mathrm{pH}$, puis après un bref épisode de réaugmentation, une nouvelle décroissance amenant le pH à une valeur moyenne de 2,0. Le second repas d'aliment $B$ (6I $g$ ingérés en moyenne) n'induit pour ce premier type de porcelets qu'une augmentation très progressive $\mathrm{du} \mathrm{pH}$ intragastrique qui atteint alors en $30 \mathrm{mn}$ environ une valeur maximum de 4,0 .

Chez les porcelets présentant le second type de réponse, la distribution matinale d'aliment $\mathrm{B}$ provoque des variations de la courbe de $\mathrm{pH}$ en tous points comparables à celles du premier type, hors le fait que l'augmentation du pH est encore plus rapide, et débute dès la première minute après le début du repas. Dans ce cas la distribution $\mathrm{du} 2^{\mathrm{e}}$ repas est à l'origine d'une variation de la courbe semblable à celle induite par le repas du matin. Le $\mathrm{pH}$ atteint alors chez ces porcelets des valeurs comprises entre 5,0 et 7,0 dans les minutes qui suivent le début du second repas.

\section{2. - Caractéristiques du contenu gastrique après prélèvements}

a) $p H$ et activité peptique des échantillons prélevés.

L,a valeur moyenne du $\mathrm{pH}$ mesuré sur les prélèvements de contenu gastrique pour tous les animaux âgés de 18 à 25 jours est présentée dans le tableau 3. Sur la base de ces valeurs moyennes, il apparaît que l'évolution du $\mathrm{pH}$ du contenu est analogue pour les 2 aliments et les 2 repas. De plus, l'examen des valeurs moyennes recueillies, quel que soit l'aliment, mais en fonction de l'âge des porcelets (fig. 4) montre l'absence de modification notable entre 18 et $3 \mathrm{I}$ jours.

En ce qui concerne l'activité peptique des contenus frais de l'estomac entre I 8 et 25 jours (tab1. 3) on n'observe aucune influence marquante de la nature de l'aliment ou de l'heure de distribution du repas sur les valeurs moyennes. En revanche une nette influence de l'âge est observée vis-à-vis de l'activité peptique du contenu gastrique résiduel prélevé avant les repas (fig. 5). Mais cette différence est abolie sur les échantillons recueillis $\mathrm{I} h$ après le repas.

I,absence précédemment constatée de différences systématiques des effets du repas selon l'heure de distribution vis-à-vis du $\mathrm{pH}$ ou de l'activité peptique, autorise un regroupement des valeurs moyennes pour les 2 repas de la journée (fig. 6). Ce mode de présentation souligne les variations inverses du $\mathrm{pH}$ et de l'activité peptique lors du repas, quel que soit l'aliment distribué. 


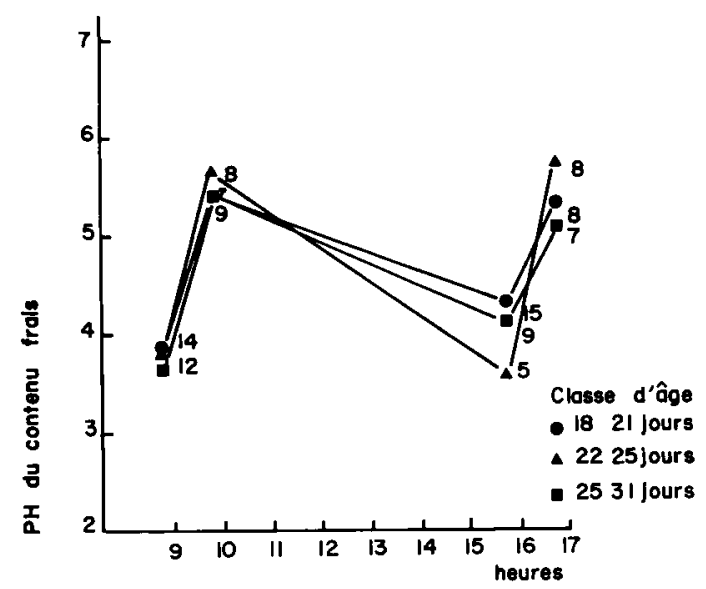

FIG. 4. - Valeurs moyennes par classe d'âge $d u p H$ du contenu gastrique frais selon l'heure Repas à $9 \mathrm{~h}$ et $\mathrm{I} 6 \mathrm{~h}$

\section{TABLEAU 3}

Consommation alimentaire des porcelets soumis aux prélèvements de contenu gastrique $p H$ et activité peptique des contenus gastriques prélevés selon l'heure du repas et la nature de l'aliment

$\bar{x}:$ valeurs moyennes

$s$ : écart-type de la population

\begin{tabular}{|c|c|c|c|c|c|c|c|c|}
\hline & \multirow{2}{*}{\multicolumn{2}{|c|}{ Aliment }} & \multicolumn{2}{|c|}{ Repas $9 \mathrm{~h}$} & \multicolumn{2}{|c|}{ Repas $16 \mathrm{~h}$} & \multicolumn{2}{|c|}{2 repas confondus } \\
\hline & & & Avant & $1 \mathrm{~h}$ après & Avant & $1 \mathrm{~h}$ après & Avant & $1 \mathrm{~h}$ après \\
\hline \multirow{2}{*}{$\begin{array}{l}\text { Quantité d'aliment } \\
\text { consommée (en g) }\end{array}$} & A & $\begin{array}{l}\bar{x} \\
s\end{array}$ & $\begin{array}{l}51,8 \\
10,4\end{array}$ & $\begin{array}{l}49,8 \\
28,0\end{array}$ & $\begin{array}{l}48,6 \\
13,9\end{array}$ & $\begin{array}{l}44,1 \\
15,8\end{array}$ & $\overline{-}$ & - \\
\hline & B & $\begin{array}{l}\bar{x} \\
s\end{array}$ & $\begin{array}{l}57,5 \\
25,4\end{array}$ & $\begin{array}{l}36,4 \\
17,9\end{array}$ & $\begin{array}{l}42,6 \\
21,9\end{array}$ & $\begin{array}{l}37,3 \\
18,4\end{array}$ & - & - \\
\hline \multirow{2}{*}{$\begin{array}{l}\text { pH du contenu } \\
\text { gastrique brut }\end{array}$} & A & $\begin{array}{l}\bar{x} \\
s\end{array}$ & $\begin{array}{l}3,96 \\
0,74\end{array}$ & $\begin{array}{l}5,50 \\
0,39\end{array}$ & $\begin{array}{l}4,08 \\
0,61\end{array}$ & $\begin{array}{l}5,56 \\
0,43\end{array}$ & $\begin{array}{r}4,01 \\
0,67 \\
n=23\end{array}$ & $\begin{array}{c}5,53 \\
0,40 \\
n=16\end{array}$ \\
\hline & B & $\begin{array}{l}\bar{x} \\
s\end{array}$ & $\begin{array}{l}3,67 \\
1,36\end{array}$ & $\begin{array}{l}5,63 \\
0,40\end{array}$ & $\begin{array}{l}4,17 \\
0,79\end{array}$ & $\begin{array}{l}5,49 \\
0,58\end{array}$ & $\begin{array}{r}3,89 \\
1,03 \\
n=23\end{array}$ & $\begin{array}{r}5,57 \\
0,48 \\
n=17\end{array}$ \\
\hline \multirow{2}{*}{$\begin{array}{c}\text { Activité peptique } \\
\text { par } \mathrm{g} \\
\text { de contenu frais }\end{array}$} & A & $\begin{array}{l}\bar{x} \\
s\end{array}$ & $\begin{array}{l}410 \\
372\end{array}$ & $\begin{array}{l}131 \\
105\end{array}$ & $\begin{array}{l}365 \\
206\end{array}$ & $\begin{array}{l}92 \\
44\end{array}$ & $\begin{array}{l}392 \\
302 \\
n=10\end{array}$ & $\begin{array}{c}110 \\
76 \\
n=11\end{array}$ \\
\hline & B & $\begin{array}{l}\bar{x} \\
s\end{array}$ & $\begin{array}{l}358 \\
246\end{array}$ & $\begin{array}{l}53 \\
16\end{array}$ & $\begin{array}{l}439 \\
343\end{array}$ & $\begin{array}{l}67 \\
23\end{array}$ & $\begin{array}{l}389 \\
276 \\
n=13\end{array}$ & $\begin{array}{c}60 \\
20 \\
n=10\end{array}$ \\
\hline
\end{tabular}






FIG. 5. - Valeurs moyennes par classe d'âge de l'activité peptique du contenu gastrique homogénéisé selon l'heure Repas à 9 h et $16 \mathrm{~h}$

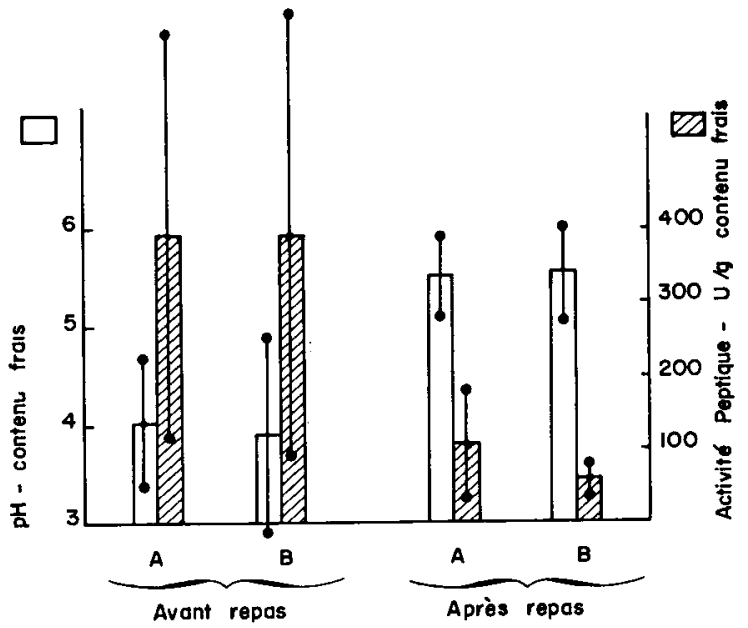

Fig. 6. - Influence du repas et de la composition de l'aliment sur les valeurs moyennes du $p H$ et de l'activité peptique des contenus gastriques chez des porcelets ágés de 18 à 25 jours avec indication de l'écart-type de la population de données 
B. SÈVE, J.-P. IAPLACE

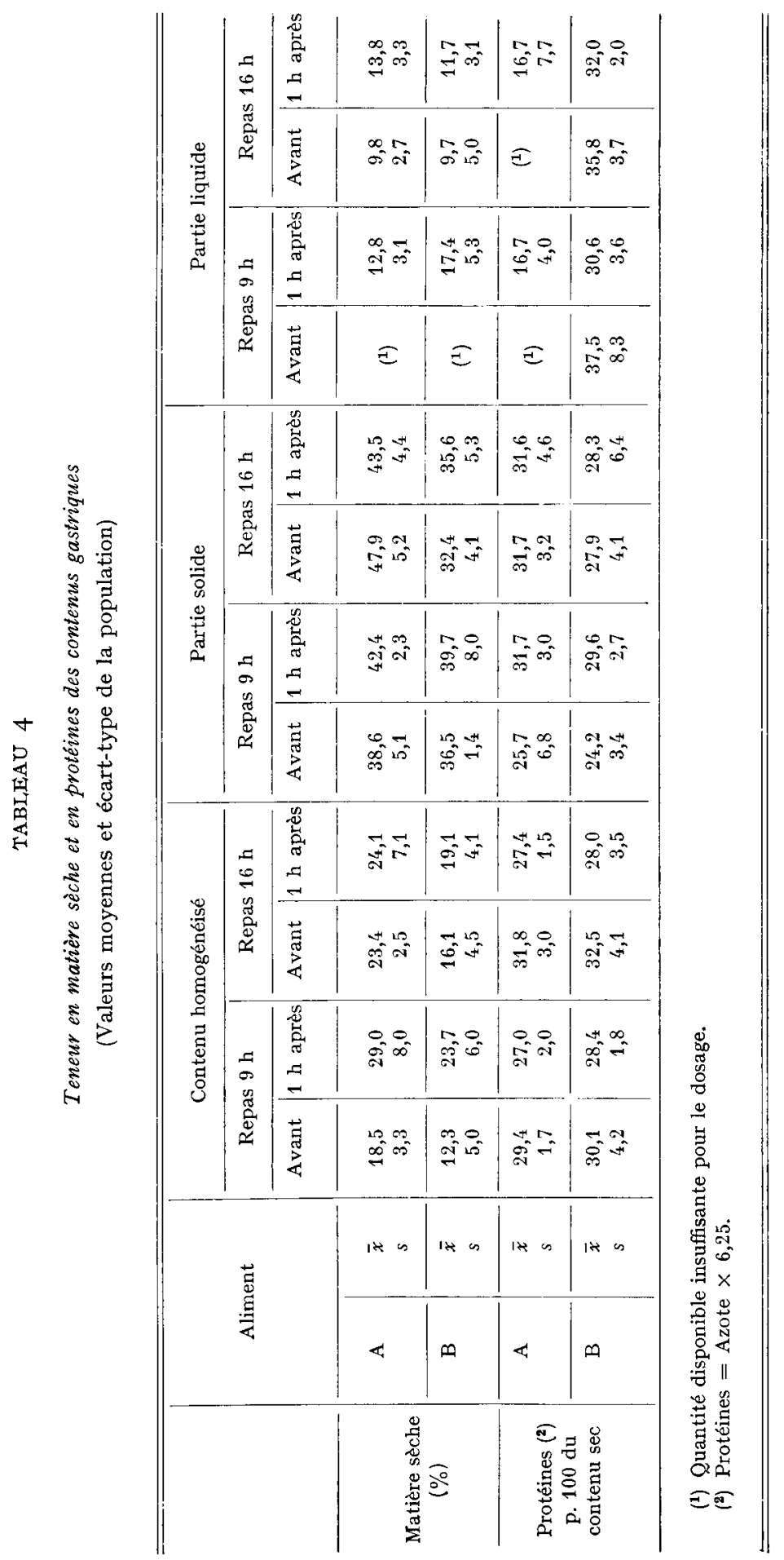


b) Teneurs en matière sèche et azote des contenus gastriques.

Les teneurs moyennes en matière sèche et azote du contenu gastrique homogénéisé et de ses fractions liquide et solide sont présentées dans le tableau 4 après regroupement des animaux indépendamment de 1'âge qui n'introđuit pas de variation de ces paramètres.

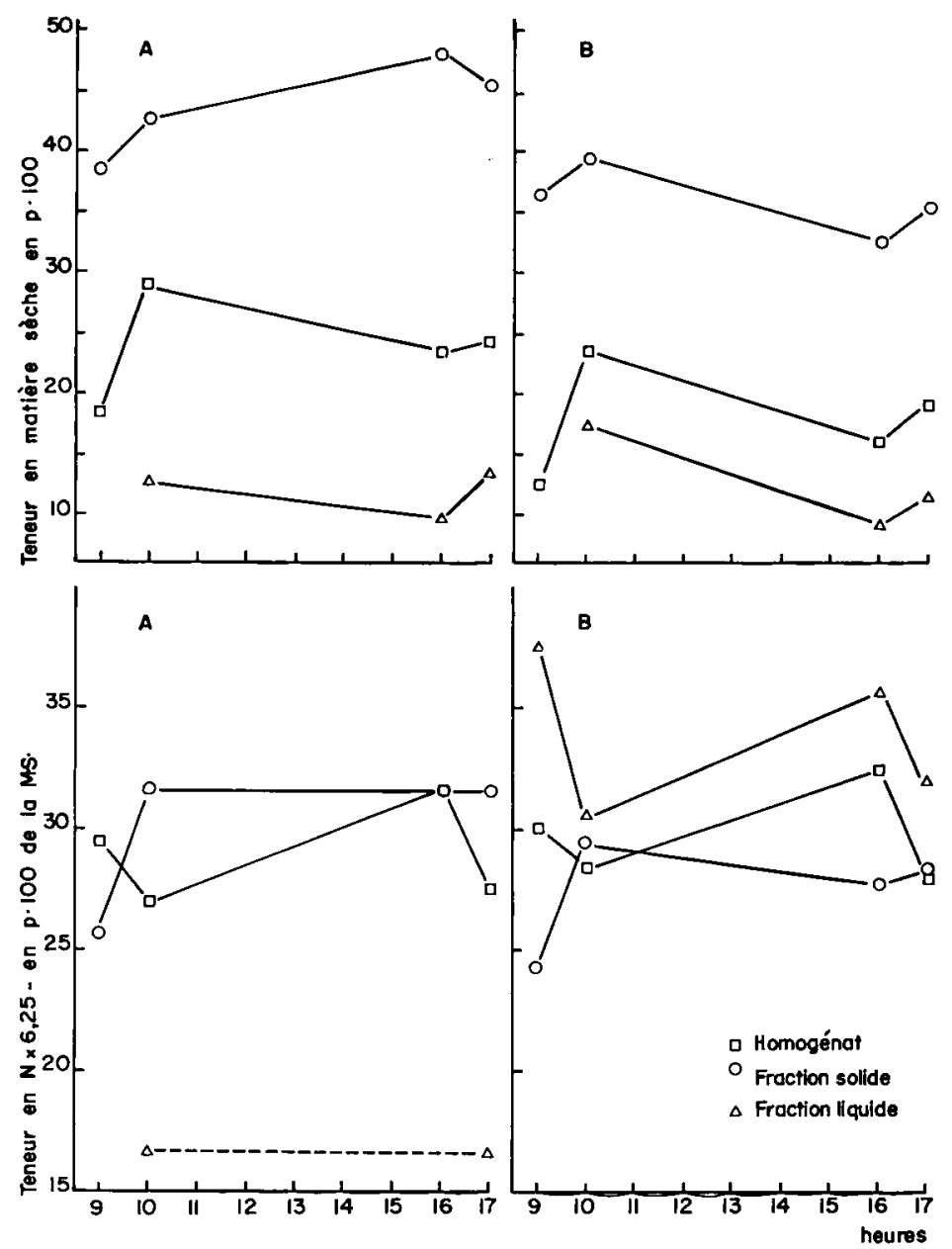

FIG. 7. - Influence du repas et de la composition de l'aliment

sur la teneur en matière sèche du contenu gastrique frais et sur la teneur en azote du contenu sec

Dans le cas d'ingestion d'aliment $A$, la teneur en matière sèche du contenu homogénéisé augmente fortement lors du premier repas, alors qu'elle ne croît que faiblement dans un estomac incomplètement évacué lors du $2^{\mathrm{e}}$ repas. Celui-ci survient à un stade où la teneur en matière sèche reste encore nettement supérieure à celle enregistrée avant le repas du matin. Si l'on examine l'évolution de la fraction coagulée du contenu gastrique, il apparaît que la teneur en matière sèche 
croît depuis le début du repas du matin jusqu'au début du repas de $I 6$ h. Ce dernier induit en $I h$ une réduction rapide jusqu'à un niveau comparable à celui mesuré I $\mathrm{h}$ après le début du repas matinal. Une variation de sens inverse est observée lors du $2^{\text {e }}$ repas pour la fraction liquide.

La teneur en azote du contenu gastrique sec, lors d'ingestion de ce même aliment $\mathrm{A}$, n'est que très faiblement affectée en ce qui concerne l'homogénat. Dans la fraction coagulée sèche, cette teneur croît lors du repas matinal mais reste ensuite stationnaire, et environ deux fois plus élevée que dans la matières sèche de la fraction liquide. L'illustration de toutes ces variations est présentée dans la figure 7 parallèlement aux effets du régime $B$.

Les modifications observées chez les porcelets ingérant l'aliment $B$ ne diffèrent de celles précédemment décrites que pour la teneur en matière sèche de la fraction coagulée et pour la teneur en azote de la fraction liquide du contenu gastrique. En effet, la teneur en matière sèche du coagulum, augmentée dans l'heure qui suit chacun des 2 repas, décroît considérablement dans l'intervalle ro $\mathrm{h}$ à $\mathbf{I} 6 \mathrm{~h}$. La teneur en azote de la phase liquide, réduite dans l'heure qui suit chaque repas en particulier le matin, augmente régulièrement dans l'intervalle so h-I6 h.

\section{DISCUSSION}

Le relèvement rapide du $\mathrm{pH}$ intragastrique lors de la première distribution d'aliment est un phénomène constamment observé chez le Porcelet (MANER et al., i962 ; BraUde, NewPort et Porter, I970; Schulman, I973) comme chez le Porc en croissance finition (LAPLACE, I974). Dans le cas du porcelet, cette augmentation existe quelle que soit la nature du régime (caséine-lactose, lait de vache, lait de truie, lait de soja, aliment de sevrage classique). Cependant, l'amplitude de cette variation d'une part, l'évolution du $\mathrm{pH}$ au-delà de cette phase d'autre part font l'objet de descriptions variables.

Les divergences quant à l'amplitude de cette variation résultent, non de la valeur maximum atteinte (de l'ordre de 5,0 à 6,0), mais du pH initial avant le repas matinal. WALKER (I959) observe pour des porcelets en allaitement maternel exclusif et par une technique d'abattage des valeurs comprises le plus souvent entre 3 et $4,2 \mathrm{~h}$ après la dernière tétée, soit peu avant la tétée suivante. De même pout des porcelets allaités, sacrifiés ro mn avant une tétée, ScrurmaN (I973) enregistre aussi des valeurs de l'ordre de 3 à 4 .

Pour des porcelets alimentés au lait de vache (sous forme liquide) et à jeûn depuis 3 h, BraUde, NewPORT et PORTER (I970) mesurent un pH de 4,o. En revanche, MANER et al. (Ig6z) chez des porcelets de 4 semaines porteurs d'une fistule gastrique et pour des régimes à base de caséine ou de protéines de soja, constatent des $\mathrm{pH}$ moyens de $I, 6$ et $I, 8$, valeurs qui sont plus proches de celles que nous avons obtenues pour des aliments secs de remplacement du lait maternel chez des porcelets fistulés. Deux facteurs essentiels paraissent susceptibles d'éclairer cette variabilité : animaux allaités ou sevrés; technique d'abattage ou fistulation. L'influence de la présentation de l'aliment (liquide ou sec) peut également être en cause, par l'intermédiaire de l'abreuvement et des sécrétions digestives (salivaire et gastrique). 
Dans le cas de nos résultats, on peut remarquer que les valeurs moyennes du $\mathrm{pH}$ relevées avant le repas matinal sont plus élevées lors de mesure sur un prélèvement $\mathrm{du}$ contenu que lors d'enregistrement in vivo. Cette influence de la méthodologie ressort également des observations de MANER et al. (I962) qui constatent une élévation du $\mathrm{pH}$ de 2 unités entre la mesure in vivo et la mesure sur le contenu gastrique prélevé à l'abattage.

En ce qui concerne l'évolution du $\mathrm{pH}$ au cours de la journée après l'augmentation matinale, nous avons constaté avec l'aliment A (à base de lait) une décroissance relativement rapide suivie d'un plateau, aspect très voisin de celui décrit par MANER et al. (I962) pour un régime à base de caséine. De même l'évolution décrite par ces auteurs pour l'aliment à base de protéines de soja, plus lente, présente une certaine analogie avec la décroissance observée pour l'aliment B. L'amplitude de la décroissance est cependant plus faible pour les aliments étudiés. Par contre, l'effet du second repas qui ne fait pratiquement jamais l'objet de description dans la littérature est très différent selon l'aliment : alors que le $\mathrm{pH}$ n'est pas modifié dans le cas du régime $A$, il augmente toujours, quoique de façon variable selon les animaux, pour le régime $B$. Cette évolution caractéristique du régime pour le repas de l'aprèsmidi, observée chez le Porcelet, rappelle la différence très marquée observée chez des porcs de $60 \mathrm{~kg}$ environ pour 2 régimes de composition distincte (LAPLACE, I974).

Les données concernant l'activité peptique sont difficilement comparables entre auteurs en raison de différences dans les techniques ou les substrats utilisés pour cette mesure. BRAUdE, NEWPORT et PORTER (I970) notent que l'activité peptique totale du contenu gastrique croît lors du repas, alors que l'activité par gramme de contenu paraît plus élevée avant le repas. Chez nos animaux, l'activité peptique par gramme de contenu frais est effectivement beaucoup plus élevée avant les repas qu' $I$ h après. Mais elle ne concerne évidemment qu'une faible quantité de contenu avant l'ingestion alimentaire. Un autre phénomène particulièrement intéressant est décelé : cette activité est d'autant plus élevée que l'animal est plus âgé. Mais cet effet est masqué à la suite du repas. Il est possible que le faible contenu résiduel avant le repas présente des caractéristiques très voisines de celles décrites par LEWIS et al. (I957) pour le tissu gastrique sec et confirmées par BRAUDE et al. (I958).

Les résultats obtenus quant à la teneur en matière sèche du contenu gastrique présentent un intérêt particulier en ce qui concerne la phase solide. En effet, la teneur en matière sèche de cette fraction augmente entre les repas d'aliment $A$ alors qu'elle décroît entre les repas d'aliment B. Cette particularité peut être interprétée comme le reflet d'une contraction du coagulum très dense pour l'aliment $\mathrm{A}$ à base de lait de vache et d'une liquéfaction progressive du floculat beaucoup plus fragmenté que donne l'aliment $B$. I a teneur en azote du contenu gastrique sec chez nos porcelets recevant l'aliment $A$, riche en caséine, pourrait être éventuellement comparée aux données de BrAUde, NEWPORT et PORTER (I970) mais celles-ci sont obtenues pour une alimentation liquide (lait de vache homogénéisé) alors que nos porcelets reçoivent un aliment sec. De plus, le mode de séparation des fractions solide et liquide est différent (centrifugation VS. filtration).

La teneur en matière sèche du contenu et la teneur en azote de celle-ci permettent d'estimer, quelle que soit 1'heure du repas, la proportion d'azote présent dans chacune des 2 phases du contenu gastrique. Ce calcul montre que $70 \mathrm{p}$. Ioo de l'azote se trouve dans la fraction solide $\mathrm{I} h$ après le début du repas d'aliment $\mathrm{A}$ alors que $30 \mathrm{p}$. Ioo 
seulement de l'azote sont retrouvés dans cette même fraction pour l'aliment $B$. Cette constatation, associée à celle relative à la texture de la fraction solide et à sa désagrégation, conduit à conclure que la matière sèche d'une manière générale et l'azote en particulier peuvent être beaucoup plus rapidement évacués vers l'intestin grêle dans le cas de l'aliment $B$. Une vacuité gastrique relative plus précoce après le repas du matin, associée à l'effet de plus grandes quantités de produits de la digestion sur les zones réceptrices du duodénum au cours des premières heures de la digestion, peut expliquer l'aspect très différent de la courbe de $\mathrm{pH}$ enregistrée lors du second repas.

Parmi les aliments de remplacement utilisables pour le sevrage précoce du porcelet, l'aliment B ici étudié offre l'avantage d'une " facilitation " digestive dans la mesure où la matière sèche et notamment l'azote sont plus rapidement disponibles pour l'absorption. Il convient cependant de souligner l'importance de la nature du substitut du lait (protéine et glucide) chez un jeune porcelet dont la fonction pancréatique n'a pas atteint son plein développement (Aumaitre, I972). Pour cette raison, il n'est peut être pas souhaitable de chercher à abréger plus la digestion gastrique des substituts du lait maternel. Une confirmation indirecte de cette conception est apportée par les résultats de digestibilité et de croissance obtenus avec incorporation de quantités croissantes de protéines solubles de poisson dans 1'aliment (SEVE, Aumaitre et Tord, I975). En effet, dans la périođe 12 à 42 jours d'âge, la meilleure adaptation digestive et la meilleure croissance sont obtenues pour l'incorporation de protéines solubles de poisson à raison de $33 \mathrm{p}$. roo des protéines totales.

\section{CONCLUSIONS}

Chez le Porcelet sevré à I2 jours et âgé de 18 à $3^{\circ}$ jours, la composition du substitut du lait maternel exerce une influence sur l'évolution du $\mathrm{pH}$ intragastrique au cours de la journée, et sur les caractéristiques respectives des phases solide et liquide du contenu gastrique. Aucune influence de la composition de 1'aliment n'est décelée sur l'activité peptique du contenu gastrique, qui croît par ailleurs avec l'âge.

L'ensemble des résultats tend à montrer que le remplacement des protéines de lait de vache par des protéines solubles de poisson, dans les limites choisies, entraîne une accélération de la digestion gastrique dont l'intérêt est une présentation plus rapide des protéines à la digestion intestinale.

L'avantage de ce phénomène d'accélération lié au remplacement d'une fraction (mais d'une fraction seulement) des protéines du lait par des protéines solubles de poisson, se traduit par l'amélioration des performances des porcelets. L'intérêt zootechnique d'une telle substitution résulte donc à la fois de l'avantage économique (coût des protéines de poisson) et d'une meilleure adaptation de cet aliment de remplacement à l'état physiologique du porcelet soumis au sevrage précoce. 


\author{
SUMMARY \\ INFLUENCE OF THE COMPOSITION OF MILK REPLACERS \\ ON SOME CHARACTERISTICS OF THE GASTRIC CONTENT \\ IN PIGLETS WEANED AT I 2 DAYS
}

A gastric fistulation in the fundic region was made in 19 Large White piglets, weaned at the age of $\mathrm{I} 2$ days, operated at $\mathrm{I}_{4.5}$ days and weighing on an average $4.0 \mathrm{~kg}$. The intragastric $\mathrm{pH}$ was recorded in vivo during diurnal periods of 7 hours. Samplings of gastric content, made before and $I$ hour after the two main meals, allowed measurement of $\mathrm{pH}$ and peptic activity in the homogenized contents. The amount of dry matter in the gastric content and that of nitrogen in the dry content were measured on the homogenate, the solid and liquid fractions being determined separately.

According to the diet used (substitution or not of $4^{8}$ p. I 00 milk protein by soluble fish protein), the curves of evolution of $\mathrm{pH}$ in vivo exhibited particular shapes. The peptic activity, increasing with age, was not affected by the composition of the diet. The distribution of dry matter and nitrogen between the solid and liquid phases of the gastric content showed that the proportion of nitrogen immobilized in the solid phase I hour after ingestion of the milk diet represented 70 p. Ioo versus only $30 \mathrm{p}$. roo in the case of the diet containing soluble fish protein. The impact of these data is discussed in relation with the improvement of performances in early weaned piglets.

\title{
RÉFÉRENCES BIBLIOGRAPHIQUES
}

Asson M. L., I938. The extinction of pepsin, trypsin, papain and cathepsin with hemoglobin. $J$. Gen. Physiol., 22, 79.

Aumaitre A., I965. Bases physiologiques du sevrage du Porcelet. Alimentation Vie, 53, r60-177.

Aumaitre A., I972. Development of enzyme activity in the digestive tract of the suckling pig : Nutrition significance and implications for weaning. Wld. Rev. Anim. Prod., 8, 54-68.

Braude R., Dollar A. M., Mrrchell K. G., Porter J. W. G., Walker D. M., I958. Proteolytic enzymes and the clotting of milk in the stomach of the young pigs. Proc. Nutr. Soc., 17, XLIX.

Braude R., Newport M. J., Porter J. W. G., r97o. Artificial rearing of pigs. II. The time course of milk protein digestion and proteolytic enzyme secretion in the 28 day old pig. Br. $J . N u t r ., 24$, $827-842$.

Hartman P. A., Hays V. W., Baker R. O., Neagle R. H., Catron D. V., ig6i. Digestive enzyme development in the young pig. J. anim. Sci., 20, II4-123.

Hill K. J., Noakes D. E., Lowe R. A., I969. Gastric digestive physiology of the calf and piglet. In : Phillipson A. T., Physiology of digestion and metabolism in the ruminant. Proc. $3^{\text {rd }}$ Int. Symp., Cambridge, I66-I79, Oriel Press Ltd, Newcastle upon Tyne.

LAPLACE J. P., I974. Enregistrement du pH intragastrique chez le Porc: variations liées à la nature, à l'importance et à l'intervalle des repas chez l'animal en finition. Ann. Zootech., 23, 89-ro4.

Lewis C. J., Hartman P. A., Liu C. H., Baker R. O., Catron D. V., i957. Digestive enzymes of the baby pig. Pepsin and trypsin. Agr. Food Chem., 5, 687-69o.

Maner J. A., Pond W. G., Loosli J. K., Lowrex R. S., I962. Effect of isolated soybean protein and casein on the gastric $\mathrm{pH}$ and rate of passage of food residues in baby pigs. J. Anim.Sci., 21, 49-53.

Mathieu C.-M., I968. Étude de la vidange stomacale du lait entier chez le Veau préruminant. $A n n$. Biol. anim. Bioch. Biophys., 8, 581-583.

Seve B., Aumaitre A., Tord P., I975. Valeur alimentaire de farines solubles de poisson blanc préparées selon divers procédés technologiques. Essais d'incorporation aux aliments d'allaitement artificiel du porcelet sevré à r 2 jours. Ann. Zootech., 24, 2I-42.

Schulman A., r973. Effect of weaning on the $\mathrm{pH}$ changes of the contents of the piglet stomach and duodenum. Nord. Vet. Med., 25, 220-225.

WALKER D. M., I959. The development of the digestive system of the young animal. I. Tissue weights, dry matter of tissues, total acidity and chloride content of stomach contents in the young pig. $J$. A gric. Sci., 52, 352-356.

White F., Wenham G., Sharman G. A. M., Jones A. S., Rattray E. A. S., McDonald I., I969. Stomach function in relation to a scour syndrome in the piglet. Br. $J$. Nutr., 23, 847-858. 